\title{
Synthesis of Organo-Silane Functionalized Nanocrystal Micelles and Their Self-Assembly
}

\author{
Hongyou Fan ${ }^{1,2, \star}$, Zhu Chen, C. Jeffrey Brinker ${ }^{1,2}$, Jakalyn Clawson ${ }^{1}$, and Todd Alam ${ }^{1}$ \\ ${ }^{1}$ Sandia National Laboratories, Chemical Synthesis and Nanomaterials Department, Advanced \\ Materials Laboratory, 1001 University Blvd. Albuquerque, NM 87106 \\ ${ }^{2}$ Department of Chemical and Nuclear Engineering, the University of New Mexico, Albuquerque, \\ NM 87131 .
}

\section{Supporting information: \\ 1. FTIR and ${ }^{29}$-Si CPMAS NMR spectra of gold nanocrystal micelles after cross-linking.}
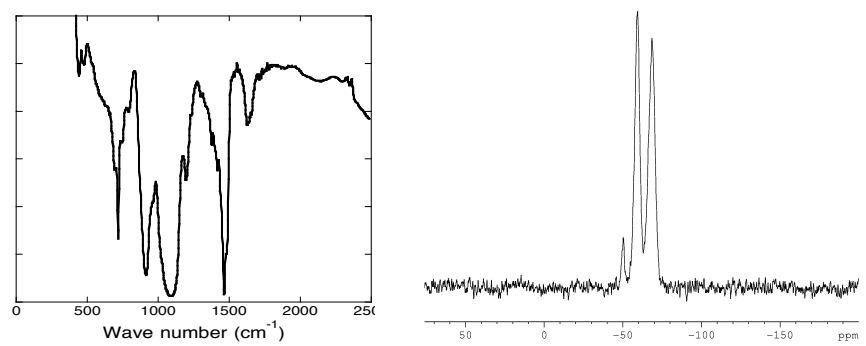

2. Synthesis of gold nanocrystals: 1-dodecanethiol derivatized gold NCs were prepared according to the method developed by Brust et al (Brust, M.; Walker, M.; Bethell, D.; Schiffrin, D. J.; Whyman, R., Journal of the Chemical Society-Chemical Communications 1994, (7), 801-802). A $60 \mathrm{ml}$ aqueous solution containing 0.7g HAuCl $\mathrm{g}_{4}$ (Aldrich) was mixed with a solution of tetraoctylammonium bromide (4g, Aldrich) in $160 \mathrm{ml}$ toluene. The two-phase mixture was vigorously stirred until the tetrachloroaurate was transferred completely into the organic layer (judged by color changes: the aqueous phase became colorless and the organic phase became dark yellow). 0.34g dodecanethiol (Aldrich) was added to the organic phase. A $40 \mathrm{ml}$ freshly prepared aqueous solution of sodium borohydride $(0.78 \mathrm{~g}$, Aldrich) was slowly added with vigorously stirring and finished during $20 \mathrm{mins}$. After further stirring for $3 \mathrm{hrs}$, the organic phase was separated and evaporated in a rotary evaporator. Heat treatment at $140^{\circ} \mathrm{C}$ was performed for 30 minutes. The gold nanocrystals were then purified by two cycles of precipitation, followed by size-selective precipitation using the solvent/nonsolvent pair of toluene/ethanol.

3. Synthesis of siloxane group functionalized gold NC-micelles: In a general preparation of water soluble gold NCmicelles, $0.58 \mathrm{~g}$ of $60 \%$ Octadecyldimethyl(3-trimethoxysilylpropyl)ammonium chloride (Gelest) methanol solution was added to 8-12g DI-water to form solution A. Solution A was stirred to completely dissolve the surfactant. $0.30 \mathrm{~g}$ 1-DT derivatized gold NCs were dissolved in 1-2 ml chloroform to form solution B. Solutions A and B were mixed together with vigorous stirring, and the chloroform was removed by quick heating to finish the encapsulation. A darkcolored solution (stock solution, C) was finally obtained and centrifuged at 2000rpm for $5 \mathrm{mins}$ to remove precipitates if any. Solution $\mathrm{C}$ can be further diluted for UV-vis spectroscopy or TEM characterization. 2-D hcp superlattice was formed upon drying of diluted stock solution $\mathrm{C}$.

4. Characterizations: The XRD spectra were used to characterized 3-d ordered arrays (film) and recorded on a Siemens D500 diffractometer using Ni-filter CuK $\alpha$ radiation with $\lambda=1.54 \AA$ in $\theta-2 \theta\left(2 \theta=1-10^{\circ}\right)$ scan mode using step size ranging from 0.02 and dwell time of 1. TEM images were taken at JEOL 2010 high-resolution microscope equipped with Gatan slow scan CCD camera and operated at $200 \mathrm{keV} .{ }^{29} \mathrm{Si}-\mathrm{NMR}$ spectra were obtained on the Bruker Avance 400 using a $4.0 \mathrm{~mm}$ MAS probe spinning at $6 \mathrm{kHz}$. Spectra were obtained using a CPMAS experiment, with at $3.0 \mu \mathrm{s} \pi / 2{ }^{1} \mathrm{H}$ pulse, $10 \mathrm{~s}$ recycle delay, a contact time of $5 \mathrm{~ms}$, and 1080 scans. Chemical shifts were externally referenced to the downfield peak of Q8M8 at $11.5 \mathrm{ppm}$. 